\title{
Analytical Approach for Air pollution Emitted From Line Source with Chemical Reaction and Wet Deposition
}

\author{
Bhaskar $\mathrm{C}^{1,2}$, Lakshminarayanachari $\mathrm{K}^{3}$, Pandurangappa $\mathrm{C}^{4}$ \\ Department of Mathematics, Sai Vidya Institute of Technology, Rajanukunte, Bengaluru- 560064, India. Email: \\ bhaskarchola@gmail.com \\ Research and Development Centre, Bharathiar University, Coimbatore-641046,India. \\ Department of Mathematics, Sai Vidya Institute of Technology, Rajanukunte, Bengaluru-560064, India. Email: \\ lnc2research@gmail.com \\ Department of Mathematics, University BDT College of Engineering, Davangere - 577004, India. Email: \\ pandurangappa_c@yahoo.co.in
}

\begin{abstract}
Wet deposition acts crucial task in the environment to supply nutrients and toxic essence to terrestrial and aquatic surroundings. Wet deposition absorbs pollutants from the environment and adds drastically to their pollutants transport. A comprehensive analytical model for the distribution of air pollutants with chemical reaction and wet deposition for line source is developed. This analytical model explains about diffusion of primary pollutants transmitted from line source in an urban area in the existence of chemical reaction and wet deposition. We have considered the large scale wind as constant and eddy diffusivity is treated as function of vertical height $\mathrm{z}$. In this paper, we used variable separable method, orthogonality condition of Bessel function and the special functions method. Chemical reaction and wet deposition escalate the diffusion of pollutants in the environment in the urban area towards right side of centre of heat Island. The pollutant concentration is less in magnitude for neutral atmosphere when compared to the stable atmosphere situation. This work reveals that pollutant concentration is less in the presence of wet deposition an urban area.
\end{abstract}

Keywords: Advection diffusion equation, Variable separable method, Mesoscale wind, Line source, Chemical reaction, Wet deposition.

\section{INTRODUCTION}

In the atmosphere the loss of aerosol particles and gases are by wet deposition. Meanwhile deposition procedure of various air contaminants can cause a variety of injuries which affects both on ecosystem and build environments. Atmospheric concentration and human health is affected by deposition of air contaminants. The serious problems one need to look at air pollution threat is the number of vehicles running on the road and the number of new vehicles entering into the road is growing exponentially. It leads to serious negative heed to the highway traffic and busy roads. Especially in developed and developing countries there is high contribution in releasing pollutants and thereby posing serious threat to living beings and their surroundings in urban areas. Several studies have reported about the connection between busy roads and a range of adverse effect on human health. Griffiths (1970) noted that in city region large scale winds is inadequate to forecast atmospheric pollutants. A .A. Marrouf et al (2015) presented the changes in advection diffusion equation by influence of eddy diffusivity, Essa, K.S.M. et al (2011) have given outline of two types of eddy diffusivities by analytically in two dimensional model. These are analytical models but they have not considered the effect of mesoscale wind, chemical reaction and wet deposition in an urban area. Dilley and Yen (1971) gave a model for pollutant distribution from a line source considering the consequences of mesoscale type wind which is evolved urban heat island. J.A.Businger et al (1971) has given Flux-Profile relationships in atmospheric suface layer. M.Vekatachalppa and Sujith kumar khan(2003) formed a comprehensive time-dependent advection diffusion numerical model due to area source by considering eddy diffusivity, variable wind speed and chemically reactive nature pollutants. CM Suresha et al (2013) studied area sourcce gravitational settling velocity of pollutants and leakagenvelocity not including removal of substances by wet deposition. Lakshminarayanachari. $\mathrm{K}$ et al (2013) demonstrated a numerical model of chemically reactive nature pollutants and mesoscale type wind by area source. However these works are not considered the effect of wet deposition and chemical reaction for the line source. In view of this, we construct an analytical model for the distribution of air contaminants 


\section{International Journal of Research in Advent Technology, Vol.7, No.5, May 2019 E-ISSN: 2321-9637 \\ Available online at www.ijrat.org}

released from line source in the existence of chemical reaction and wet deposition generated by urban heat island.

\section{MATHEMATICAL MODEL FORMULATION}

The governing equation describing pollutants distribution in the atmosphere based on the gradient transport theory is

$$
\begin{gathered}
\frac{\partial C}{\partial t}+u \frac{\partial C}{\partial x}+v \frac{\partial C}{\partial y}+w \frac{\partial C}{\partial z}=\frac{\partial}{\partial x}\left(k_{x} \frac{\partial C}{\partial x}\right)+ \\
\frac{\partial}{\partial y}\left(k_{y} \frac{\partial C}{\partial y}\right)+\frac{\partial}{\partial z}\left(k_{z} \frac{\partial C}{\partial z}\right)-\left(k+k_{w}\right) C
\end{gathered}
$$

Where $u, v, w$ are the components of wind and $k_{x}, k_{y}, k_{z}$ are coefficients of eddy diffusivity terms in $x, y, z$ directions respectively, $\mathrm{C}$ is the air pollutant concentration in the atmosphere. $k$ is the coefficient of chemical reaction rate and $k_{w}$ is the wet deposition of air pollutants.

The physical problem consists of line source elevated at a distance $z_{s}=0.5$ meters from the ground. The air pollutants are transported horizontally by large scale wind and horizontally as well as vertically by the local wind called mesoscale wind. The centre of heat island is kept at origin. The concentration dispersion was computed up to the preferred downwind length $l=5 \mathrm{~km}$ i.e. $0 \leq x \leq l$.

While forming the mathematical model the sub sequent assumptions are formed:

i) We have considered the steady state condition, i.e., $\frac{\partial C}{\partial t}$ $=0$.

ii) Perpendicular velocity is much lesser than the horizontal one in $X$-direction, we ignore term $w \frac{\partial C}{\partial z}$.

iii) $x$-axis is leaning in the way of mean wind $u=U$ and $\mathrm{U}$ much larger than wind speed $\mathrm{v}$

in $y$-direction, therefore the term $v \frac{\partial C}{\partial y}$ is ignored.

From the above mentioned assumptions the basic equation (1) reduces to the form.
$U \frac{\partial C}{\partial x}=\frac{\partial}{\partial x}\left(k_{x} \frac{\partial C}{\partial x}\right)+\frac{\partial}{\partial y}\left(k_{y} \frac{\partial C}{\partial y}\right)+\frac{\partial}{\partial z}\left(k_{z} \frac{\partial C}{\partial z}\right)-$ $\left(k+k_{w}\right) C$

The advection term in horizontal path is larger than the diffusion in $\mathrm{x}$ path then we will ignore the term

$\frac{\partial}{\partial x}\left(k_{x} \frac{\partial C}{\partial x}\right)$

$U \frac{\partial C}{\partial x}=\frac{\partial}{\partial y}\left(k_{y} \frac{\partial C}{\partial y}\right)+\frac{\partial}{\partial z}\left(k_{z} \frac{\partial C}{\partial z}\right)-\left(k+k_{w}\right) C$

$\mathrm{U}$ is the component of wind velocity in the $x$ direction which varies with the downwind distance. Equation (3) is simplified with the given boundary conditions. This is assumed to be perfectly total absorption i.e.,

$$
\frac{\partial C}{\partial z}=0 \text { at } z=0, z=h \text {. }
$$

(4)

The pollutant diffusion completely through the top of the mixed layer positioned at height $\mathrm{h}$, i.e $C(x, y, z)=0$ at $z=h$

(5)

Q is the continuous line source strength which is considered as $\left(0, y, z_{s}\right)$

$U C=Q \delta\left(z-z_{S}\right)$ as $x=0$

(6)

Where $\delta$ is the Dirac delta function.

The concentration reduces to zero, far away from the source i.e

$C \rightarrow 0$ as $x, y$ and $z \rightarrow \infty$

(7)

\section{METHOD OF SOLUTION}

From Equation (3), $\mathrm{U}$ is the constant wind speed

$U=k_{0} u_{*}(1-a x)$

And $k_{z}$ is the diffusivity term expressed in terms of power law as

$k_{z}=u_{1} z^{n}$

Where $k_{0}$ is Von-Karmen constant, $u_{*}$ is the friction velocity and $u_{1}$ is the turbulence intensity. 


\section{International Journal of Research in Advent Technology, Vol.7, No.5, May 2019 E-ISSN: 2321-9637 \\ Available online at www.ijrat.org}

Integrate (3) with respective toy from $(-\infty$ to $\infty)$

$k_{0} u_{*}(1-a x) \frac{\partial C_{y}}{\partial x}=\frac{\partial}{\partial z}\left(u_{1} z^{n} \frac{\partial C_{y}}{\partial z}\right)-\left(k+k_{w}\right) C_{y}$

Equation (10) written in the form of

$$
(1-a x) \frac{\partial C_{y}}{\partial x}=\frac{u_{1}}{k_{0} u_{*}} z^{n} \frac{\partial^{2} C_{y}}{\partial z^{2}}+\frac{u_{1} n}{k_{0} u_{*}} z^{n-1} \frac{\partial C_{y}}{\partial z}-\frac{\left(k+k_{w}\right) C_{y}}{k_{0} u_{*}}
$$

Equation (11) solved using the variable separable method. We consider the solution of equation (11) in the form of

$C_{y}(x, z)=X_{1}(x) \cdot Z_{1}(z)$

(12) Substitute (12) in (11) and differentiate with respective to $x$ and $z$ partially, the result of this we get two variables $X_{1}$ and $Z_{1}$ which is ordinary differential equation is as follows

$(1-a x) \frac{1}{X_{1}} \frac{d X_{1}}{d x}+\frac{\left(k+k_{w}\right)}{k_{0} u_{*}}=-\lambda^{2}$

and

$\frac{\alpha z^{n}}{Z_{1}} \frac{d^{2} Z_{1}}{d z^{2}}+\frac{\beta z^{n-1}}{Z_{1}} \frac{d Z_{1}}{d z}=-\lambda^{2}$

(14) Where $\lambda^{2}$ is constant, $\alpha=\frac{u_{1}}{k_{0} u_{*}}$ and $\beta=\frac{u_{1} n}{k_{0} u_{*}}$

For equation (13) the general solution is given below

$X_{1}(x)=\gamma(a x-1)^{\frac{k_{0} u_{*} \lambda^{2}+k+k_{W}}{k_{0} u_{*} a}}$

$(15)$

Where $\gamma$ is arbitrary constant

Equation (14) can be written in the form of

$z^{2} \frac{d^{2} Z_{1}}{d z^{2}}+n z \frac{d Z_{1}}{d z}+\frac{\lambda^{2}}{\alpha} z^{2-n} Z_{1}=0$

On altering the dependent variable $Z_{1}$ and independent variable $z$, and substituting in equation (16) we get

$Z_{1}=Z_{*}^{\frac{1-n}{2-n}} Z_{1 *}$ and $Z_{*}=z^{\frac{2-n}{2}}$

(17)

Equation (16) reduces in the form $z_{*}^{2} \frac{d^{2} Z_{1 *}}{d z_{*}^{2}}+z_{*} \frac{d Z_{1 *}}{d z_{*}}+\left(\eta^{2} z_{*}^{2}-\mu^{2}\right) Z_{1 *}=0$

Where $\eta^{2}=\frac{4 \lambda^{2}}{\alpha(2-n)^{2}}, \mu=\frac{1-n}{2-n}$

Solution of equation (14) is attained in dissimilar initial and boundary conditions as follows:

Using the boundary conditions of equation (4) and (5) in equation (12)

$\frac{\partial Z_{1}}{\partial z}=0$ at $z=0$

(19)

$Z_{1}=0$ at $z=h$

(20)

Equation (18) in the form of Bessel equation and its solution is given by

$Z_{1}=z^{\frac{1-n}{2}}\left[A J_{\mu}\left(\eta z^{\frac{2-n}{2}}\right)+B J_{-\mu}\left(\eta z^{\frac{2-n}{2}}\right)\right]$ $A$ and $B$ are constants,

in (21) $J_{\mu}, J_{-\mu}$ are the first kind of Bessel functions of order $\mu,-\mu$ correspondingly, substituting equation (19) in equation (21) yields $B=0$ and using equation (20) at $z=h$ equation (21) we get

$h^{\frac{1-n}{2}} J_{\mu}\left(\eta z^{\frac{2-n}{2}}\right)=0$

Equation (22) represents Sturm- Liouville eigen value problem which have the corresponding Eigen functions:

$Z_{1 \propto}(z)=z^{\frac{1-n}{2}} J_{\mu}\left(\eta_{\alpha} z^{\frac{2-n}{2}}\right)=0 \quad \alpha=1,2,3 \ldots \ldots \ldots \infty$.

Substitute equations (15) and (23) in equation (12) we get

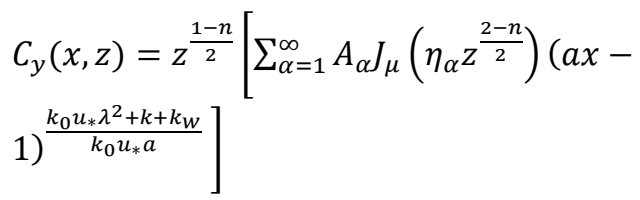

$A_{\alpha}, \alpha=1,2,3 \ldots \ldots \ldots \infty$ are the unknown parameter. Equation (24) describes the concentration distribution $C_{y}$ along the Fourier- Bessel series corresponding to a set of Eigen function $Z_{1 \alpha}$. 


\section{International Journal of Research in Advent Technology, Vol.7, No.5, May 2019 E-ISSN: 2321-9637 \\ Available online at www.ijrat.org}

Equation (6) gives in the form by evaluation of the coefficients $A_{\alpha}$ 's and source $x=0$ we get

$$
\begin{aligned}
& k_{0} u_{*} z^{\frac{1-n}{2}}(-1)^{\frac{k_{0} u_{*} \lambda^{2}+k+k_{w}}{k_{0} u_{*} a}}\left[\sum_{\alpha=1}^{\infty} A_{\alpha} J_{\mu}\left(\eta_{\alpha} z^{\frac{2-n}{2}}\right)\right]= \\
& Q_{p} \delta\left(z-z_{S}\right)
\end{aligned}
$$

To find $A_{\alpha}$ multiplying equation (24)

$$
\text { byz }{ }^{\frac{2-n}{2}} J_{\mu}\left(\eta_{\beta} z^{\frac{2-n}{2}}\right) \quad \beta \geq 0 \text { using orthogonality of }
$$

Bessel function and integrating with respective to $\mathrm{z}$ with limits 0 to $h$, we get

$$
A_{\beta}=\frac{2 Q_{p} z_{S}^{\frac{2-n}{2}}}{k_{0} u_{*} h^{2}(-1)^{\frac{k_{0} u_{*} \lambda^{2}+k+k_{w}}{k_{0} u_{*} a}}} * \frac{J_{\mu}\left(\eta_{\beta^{z_{S}}}{ }^{\frac{2-n}{2}}\right)}{J^{2}{ }_{\mu+1}\left(\eta_{\beta} h^{\frac{2-n}{2}}\right)} \quad \beta \geq 1 .
$$

Substituting $A_{\beta}$ in the equation (24), we get required solution as shown below:

$$
\begin{gathered}
C_{y}(x, z)= \\
Q_{p} \frac{2(z)^{\frac{1-n}{2}}\left(z_{S}\right)^{\frac{2-n}{2}}}{k_{0} u_{*} h^{2}}\left[\sum_{\alpha=1}^{\infty} \frac{J_{\mu}\left(\eta_{\alpha} z^{\frac{2-n}{2}}\right) J_{\mu}\left(\eta_{\alpha z_{S}} \frac{2-n}{2}\right)}{J^{2}{ }_{\mu+1}\left(\eta_{\alpha} h^{\frac{2-n}{2}}\right)}(1-\right. \\
\left.a x)^{\frac{k_{0} u_{*} \lambda^{2}+k+k_{w}}{k_{0} u_{*} a}}\right]
\end{gathered}
$$

In which $\eta_{\beta} h^{\frac{2-n}{2}}$ is given as: $J_{\mu}\left(\eta_{\beta} h^{\frac{2-n}{2}}\right)=0$

\section{RESULTS AND DISCUSSION}

In this paper, the effects of wet deposition and chemical reaction on concentration of air pollutants at the line source are analyzed. We have kept the centre of heat island at origin. The concentration circulation is calculated in the city limits up to the preferred downwind length $l=5 \mathrm{~km}$ i.e., $0 \leq x \leq l$ for stable and neutral atmospheric situations.
The figures 1(a), 1(b) gives plots of concentration verses downwind distance for vertical height $(\mathrm{z}=3 \mathrm{~m})$ in the existence and nonexistence of chemical reaction in stable and neutral atmospheric conditions. From the figures 1(a), 1(b) we find that concentration decreases as downwind distance $\mathrm{x}$ increases.It is also observed that air pollutant concentration is more near bottom surface and as the down wind distance increases the concentration decreases for both stable and neutral cases.

The figures 2(a), 2(b), 3(a), 3(b) gives plots of the variation of concentration profile verses downwind distance for different vertical heights by considering existence and nonexistence of wet depositions for stable and neutral atmospheric conditions. Here we observed that downwind distance $\mathrm{x}$ raises concentration decreases. It is also observed that the concentration of air pollutants is less $(\mathrm{z}=10 \mathrm{~m})$ when compared to near ground surface $(z=1 \mathrm{~m})$ height and as distance raises the concentration decreases in both the cases for stable and neutral conditions. Concentration of air pollutants is less in magnitude in the existence of wet deposition compared to the nonexistence of wet deposition for both stable and neutral cases.

The figures 4(a), 4(b), 5(a), 5(b) gives plots of concentration profile against vertical height for dissimilar downwind distance by considering existence and nonexistence wet deposition for stable and neutral atmospheric conditions. Here we observed that the concentration decreases as vertical height $\mathrm{z}$ increases. It is also observed that the concentration of pollutants $\mathrm{x}=$ $500 \mathrm{~m}$ is less when compared to $\mathrm{x}=100 \mathrm{~m}$ distance and as vertical height increases the concentration decreases in both cases for stable and neutral conditions. The concentration of air pollutants is maximum in magnitude in the nonexistence of wet deposition compared to the existence of wet deposition in both stable and neutral cases. 

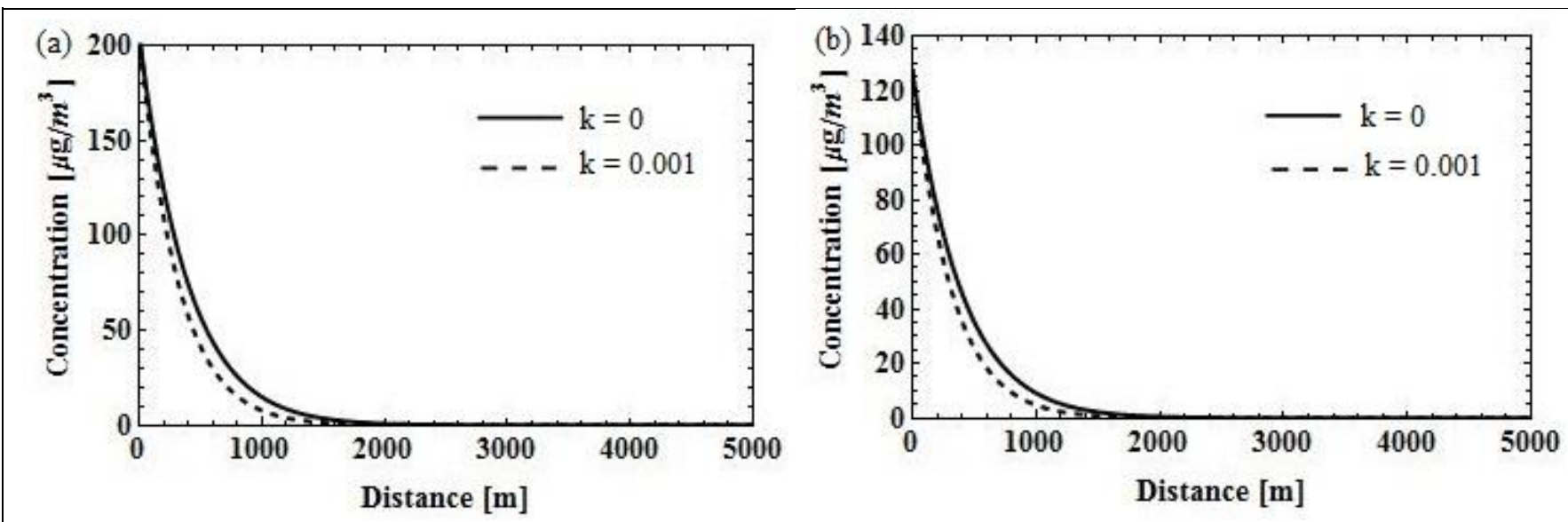

Figures 1(a), 1(b) Concentration profile versus downwind distance with and without chemical reaction at $\mathrm{z}=3 \mathrm{~m}$ for stable and nuetral atmospheric condition respectively.
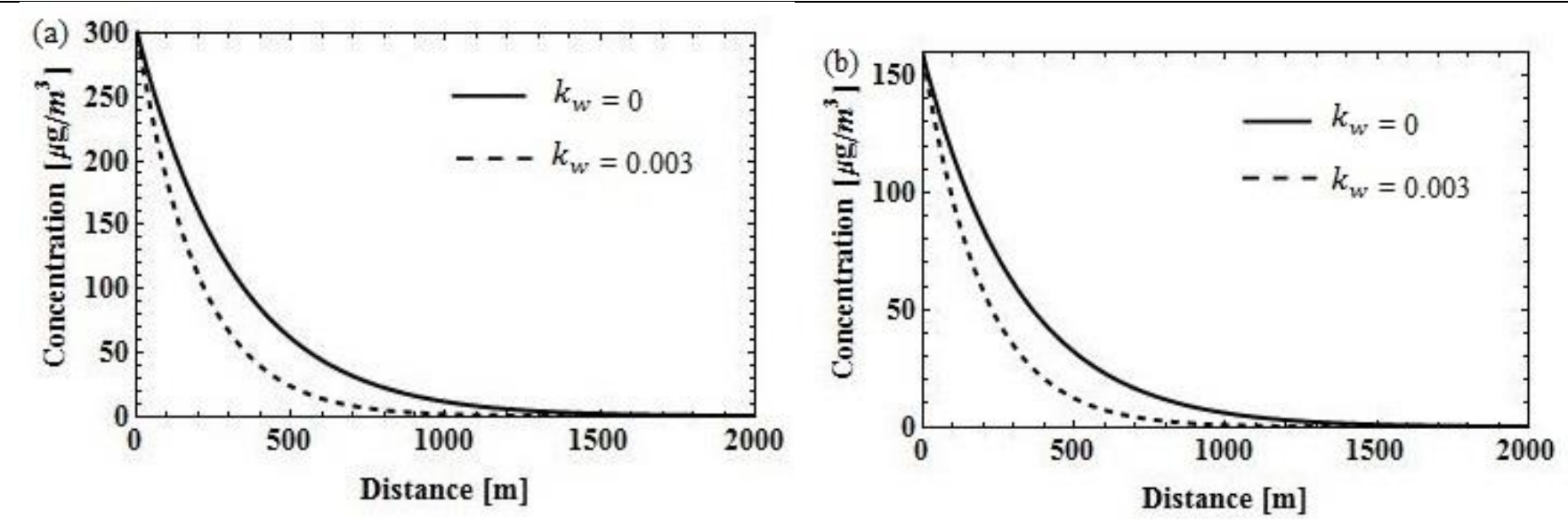

Figures 2(a), 2(b) variation of concentration profile with downwind distance for vertical heights $(\mathrm{z}=1 \mathrm{~m})$ with and without wet deposition for stable and neutral cases respectively.
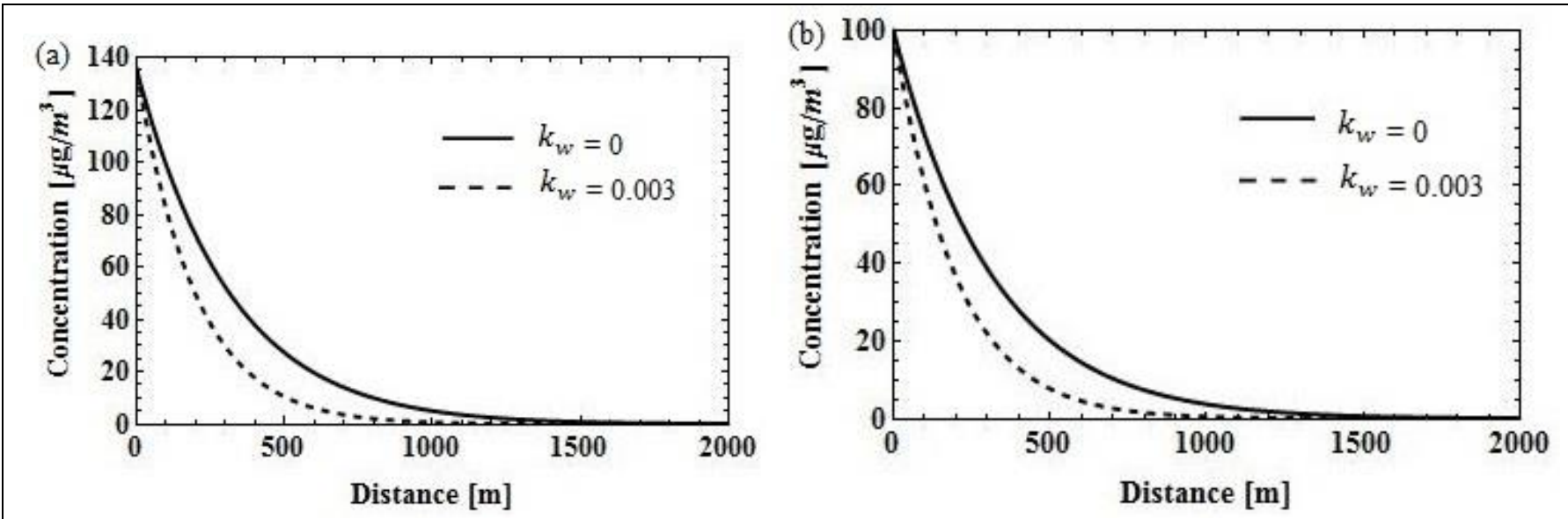
Figures 3(a),3(b) variation of concentration profile with downwind distance for vertical heights $(\mathrm{z}=10 \mathrm{~m})$ with and without wet deposition for stablel and neutral cases respectively.

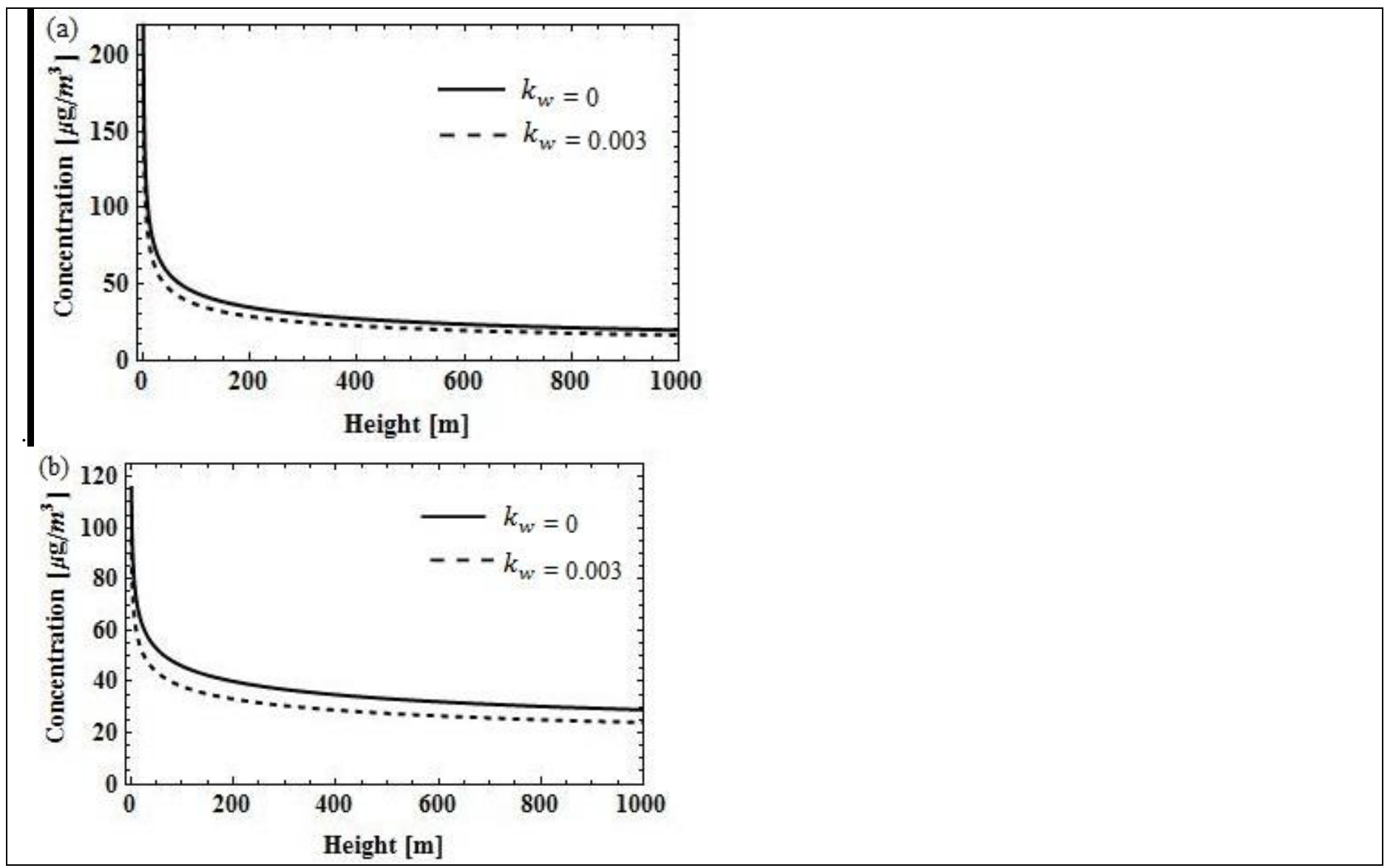

Figures 4(a),4(b) Concentration profile versus vertical height with and without wet deposition at $\mathrm{x}=100 \mathrm{~m}$ for stable and neutral atmospheric condition respectively.
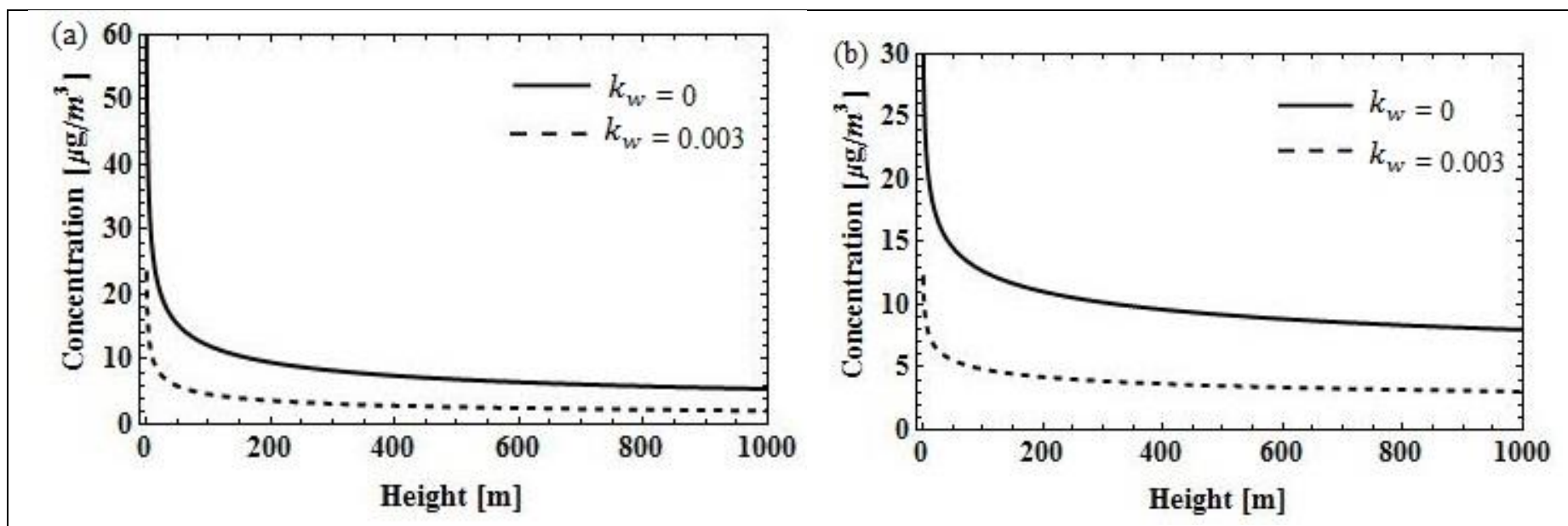

Figures 5(a),5(b) Concentration profile versus vertical height with and without wet deposition at $\mathrm{x}=500 \mathrm{~m}$ for stable and neutral atmospheric condition respectively. 


\section{International Journal of Research in Advent Technology, Vol.7, No.5, May 2019 E-ISSN: 2321-9637 \\ Available online at www.ijrat.org}

\section{CONCLUSION}

An analytical model is to calculate ambient concentration of air pollutants emitted from line source in stable and neutral conditions of atmosphere in company with the impact of chemical reaction and wet deposition is presented. The outcomes are analyzed for the dispersal of air pollutants in the downwind and vertical distances in stable and neutral conditions. This analytical model is solved using variable separable method, the Special functions technique and orthogonality property of Bessel functions. Graphs are plotted and the results are analyzed for air pollutant concentration for existence and nonexistence of wet depositions for dissimilar heights $(\mathrm{z}=1 \mathrm{~m}, \mathrm{z}=10 \mathrm{~m})$ and the downwind distance $(x=100 \mathrm{~m}, \mathrm{x}=500 \mathrm{~m})$. We perceive that when downwind distance $\mathrm{x}$ increases concentration decreases. We also perceive that downwind distance (x) and vertical height $(\mathrm{z})$ raises then concentration reduces in the existence and nonexistence of wet deposition. The amount of concentration is comparatively more in stable atmospheric condition than in neutral atmospheric condition.

\section{REFERENCES}

[1] Anjaneyulu, Y., 2002, Air Pollution And Control Technologies, allied publishers PvtLtd, India.

[2] Dilley, J. F., Yen, K. T., 1971. Effect of mesoscale type wind on the pollutant distribution from a line source. AtmosphericEnvironment,5, 843-851.

[3] Griffiths, J. F., 1970. Problems in urban air pollution in: AIAA 8th aerospace Science Meeting, AIAA, New York, 70-112.

[4] Pasquill, F. and Smith, F. B. 1983. AtmosphericDiffusion, $3^{\text {rd }}$ Edition, Wiley,Newyork.

[5] Seinfeld, J. H. 1986 Atmospheric Chemistry and Physics of Air pollution.Wiley,Newyork.

[6] Venktachalappa. M., Sujith kumar khan and khaleel Ahmed G kakamari, 2003. Time dependent mathematical model of air pollution due to area source with variable wind velocity and eddy diffusivity and chemical reaction, proc Indian Natn Sci Acad, 69A, No.6, 745-758.

[7] C. M Suresha, Lakshminarayanachari, K., Siddalinga Prasad, M., Pandurangappa, C. 2013.Numerical model of airpollutants emitted from an area source of primary and secondary pollutants with chemical reaction and gravitational settling with point sorceon the boundry. International Journal of Research in Environmental science and technology,9-18.

[8] Marrouf, A. A., Khaled S.M. Essa, Maha S. E1Otaify, Adel S. Mohamed, Galal Ismail. 2015. The
Influence of Eddy diffusivity Variation on the Atmospheric Diffusion Equation. Open Journal of Air Pollution, 04,109-118.

[9] Lakshminarayanachari, K., Sudheer Pai, K. L., Siddalinga Prasad, M., Pandurangappa, C. 2013.A Two dimensional numerical model of primary pollutant emitted from an urban area source with mesoscale, dry deposition and chemical reaction. Atmospheric Pollution Research, 4, 106-116.

[10]Essa, K. S. M., Mina, A. N and Higazy, M. 2011. Analytical solution of diffusion equation in two dimensions using two forms of eddy diffusivities, Romanian journal of physics, 56, 1228-1240.

[11] J.A.Businger,J.C.Wyngaard,Y.Izumi and E.F.Bradley,1971. Flux- Profile relation ships in atmospheric suface layer, Journal of the Atmospheric science, Volume (28),181-189 